Article

\title{
The Effect of Surface Charge on the Separation of Pyrite from Serpentine by Flotation
}

\author{
Tao Long ${ }^{1,+}$, Xiaotao Huang ${ }^{2,+}$ and Wei Xiao ${ }^{1, *}$ \\ 1 School of Resources Engineering, Xi'an University of Architecture and Technology, Xi'an 710055, China; \\ longtao@xauat.edu.cn \\ 2 The institute for advanced studies, Wuhan University, Wuhan 430072, China; huangxiaotao810@163.com \\ * Correspondence: xiaowei2015@csu.edu.cn; Tel.: +86-029-8220-3408 \\ $+\quad$ T.L. and X.H. contributed equally to this work.
}

Received: 8 September 2019; Accepted: 12 October 2019; Published: 14 October 2019

\begin{abstract}
Serpentine, a magnesium silicate mineral with positive surface charge in many sulfide ores around the world, usually deteriorates the flotation behavior by covering the target mineral surface. In this paper, the effect of surface potential regulation on serpentine flotation was revealed by flocculation experiments, zeta potential measurements, infrared spectrum analysis, and DLVO theoretical calculations. The experimental results of flocculation and sedimentation show that heterogeneous coagulation easily occurs between serpentine and pyrite particles, which reduces the floatability of pyrite. Reducing the surface potential of serpentine is an effective way to eliminate heterogeneous coagulation between minerals. The key to regulating the surface potential of serpentine is $\mathrm{Mg}^{2+}$ ion dissolution from the serpentine surface to the liquid phase. Phosphates, especially sodium hexametaphosphate, can enhance $\mathrm{Mg}^{2+}$ ion dissolution from the serpentine surface to the liquid phase and react with $\mathrm{Mg}^{2+}$ ions in the liquid phase to form stable soluble complexes.
\end{abstract}

Keywords: heterogeneous reunion; $\mathrm{Mg}^{2+}$ ion dissolution; surface potential; fine serpentine

\section{Introduction}

Serpentine is one of the most common magnesium-bearing silicate minerals in nickel/copper sulphide, rhodochrosite, and manganese hydrate ores [1-3]. When serpentine dissociates, the magnesia-oxygen octahedron layer breaks, and the ionic magnesium-oxygen bond is exposed on the surface [4]. It has strong hydration and good natural hydrophilicity. The surface hydroxyls of fine serpentine dissolve preferentially in an aqueous solution, and $\mathrm{Mg}^{2+}$ ions remain on the serpentine surface, which makes the surface charge of serpentine stronger and its point of zero charge around a $\mathrm{pH}$ of $10.0[5,6]$. In weak alkaline pulp, the surface of sulfide minerals is generally negatively charged, and fine serpentine with a positive charge is adsorbed on the sulfide mineral surface with negative charge under the action of an electrostatic adsorption force, which makes the difference in the hydrophobicity and surface properties of sulfide minerals significantly reduced and deteriorates the flotation behavior of sulfide minerals [6,7]. In view of this property of serpentine, some researchers have used serpentine as an inhibitor for the flotation separation of galena and pyrite [8-10].

The cover of fine serpentine particles on the surface of target minerals is the main factor affecting the flotation of sulfide ores by serpentine, which is mainly caused by electrostatic interaction $[6,9]$. Reducing the surface potential of serpentine and making it better dispersed in solution is the key factor to solving the problem of sulfide mineral flotation containing serpentine [11,12]. In laboratory studies, nickel pyrite was separated from serpentine by magnetic separation after encapsulating the target mineral with fine magnetic particles [13-17]. These studies were only conducted in the laboratory stage, and there are many other factors that need to be considered in magnetic package 
sorting technology from laboratory to industrial applications [17-19]. At present, most of the studies have used organic or inorganic dispersants to regulate the surface potential of serpentine in order to disperse and inhibit serpentine [5-7,11,12]. Lu et al. found that anionic components of $\mathrm{H}_{2} \mathrm{PO}_{4}{ }^{-}$ and $\mathrm{HPO}_{4}{ }^{2-}$ in sodium hexametaphosphate (SHMP) could be chemically adsorbed on the Mg sites of the serpentine surface [20]. Zhao et al. investigated the dispersive effect of low-molecular-weight sodium polyacrylate (PAAS) on pyrite-serpentine flotation and found that anionic PAAS adsorbed on the serpentine surface via electrostatic interaction [21]. Both inorganic and organic dispersants react with $\mathrm{Mg}^{2+}$ on the serpentine surface via anions to form magnesium salts that are adsorbed on the serpentine surface, thus reducing the surface potential and hydrophobicity of serpentine $[4,7,9,12]$. However, in recent studies, we found that both $\mathrm{Mg}^{2+}$ ions on the serpentine surface and dissolved $\mathrm{Mg}$ ions in solution can significantly inhibit the flotation of sulfide minerals. Therefore, it is very important to study the surface potential regulation of serpentine for the selective dispersion and inhibition of serpentine.

The purpose of this work was to reveal the regulation rule of $\mathrm{Mg}^{2+}$ ions on the serpentine surface in the mineral surface and in pulp solution. The effects of several phosphate dispersants on the surface potential of serpentine were studied by micro-flotation, microscopic observation, zeta potential measurement, solution chemical composition calculation, FT-IR analysis, and DLVO theoretical calculations. The results of this study can provide a theoretical basis for the dispersion and inhibition of serpentine in sulfide mineral flotation and for the use of serpentine as a flocculant and inhibitor.

\section{Materials and Methods}

\subsection{Materials and Reagents}

The pyrite was obtained from Yunfu, Guangdong Province, China, and the serpentine was obtained from Donghai, Jiangsu Province China. The samples were first removed by hand and then crushed in a ceramic ball 72 mill. The time was generally controlled in 3-5 min. After grinding, 200 mesh and 400 mesh sieves 73 were used for screening, more than 200 mesh sieves were returned to ceramic pots, less than 400 mesh 74 sieves were used as crushed products for zeta potential and FTIR spectroscopy testing, and 200-400 75 mesh sieves were used as flotation raw ore storage. The crystal phase composition was determined by X-ray diffraction (Germany Bruker-axes D8 Advance, Karlsruhe, Germany), the detailed processes and methods for which were previously described by Xiao [22,23]. The chemical compositions of pyrite and serpentine were determined by wavelength-dispersive $\mathrm{X}$-ray fluorescence (XRF) using an S4 Pioneer spectrometer (PANalytical B.V., Almelo, The Netherlands). The results of XRD and XRF are shown in Figure 1 and Table 1, respectively. The results demonstrate that the purities of pyrite and serpentine in the samples were calculated to be $93.9 \%$ and $83.6 \%$. The serpentine single mineral was stirred in hydrochloric acid of $2 \mathrm{~mol} / \mathrm{L}$ for $30 \mathrm{~min}$ at room temperature, filtered, washed three times with ultra-pure water, and dried in vacuum at room temperature to obtain the demagnesium serpentine sample. Trisodium phosphonate (TSP), sodium trimetaphosphate (STMP), and sodium hexametaphosphate (SHMP) of analytically pure grade were obtained from Sinopharm Chemical Reagent Co., Ltd (Shanghai, China). Ultra-pure water with a conductivity of $18.2 \mathrm{M} \Omega \cdot \mathrm{cm}$ was obtained from a USF-ELGA Maxima water purification system [24,25].

Table 1. The results of chemical composition analysis of mineral samples (wt.\%).

\begin{tabular}{cccccccc}
\hline Sample & $\mathbf{M g O}$ & $\mathrm{SiO}_{2}$ & $\mathrm{Al}_{\mathbf{2}} \mathbf{O}_{3}$ & $\mathrm{CaO}$ & $\mathrm{Fe}$ & $\mathbf{S}$ & Other \\
\hline Serpentine & 32.92 & 37.11 & 0.80 & 0.21 & 5.17 & - & - \\
Pyrite & - & - & - & - & 43.96 & 49.98 & 11.18 \\
\hline
\end{tabular}



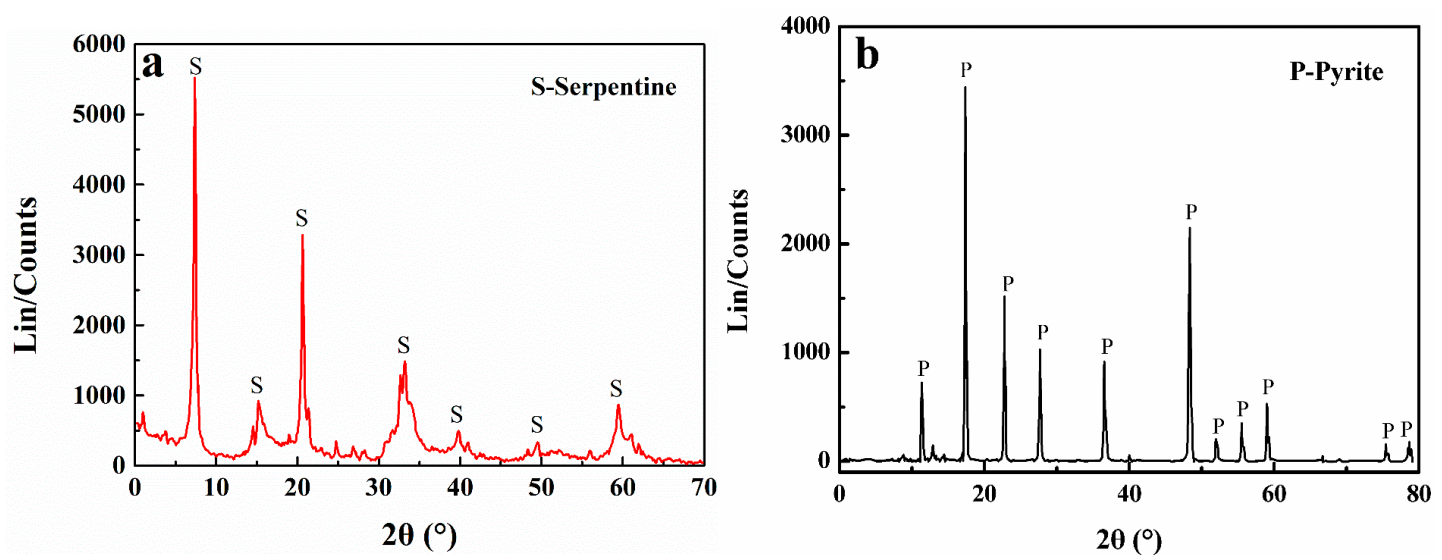

Figure 1. XRD patterns of (a) serpentine and (b) pyrite in this study.

\subsection{Methods}

\subsubsection{Micro-Flotation TESTS}

For the flotation test, we used a $40 \mathrm{~mL}$ XFG trough-type flotation machine. An ore sample weighing $2 \mathrm{~g}$ was placed in the flotation cell for each test. Then, a certain concentration of flotation reagent was added and stirred for $5 \mathrm{~min}$. The $\mathrm{pH}$ value of the slurry was measured using a precision $\mathrm{pH}$ meter. The flotation process was carried out by manual scraping, and the flotation time was 5 min. The obtained foam product and the product in the tank were dried and weighed, and the yield was calculated. For the single-mineral test, we took the flotation recovery rate to be equal to the yield; in the artificial mixed ore flotation test, the flotation recovery rate was calculated after chemical analysis of each product.

\subsubsection{Settlement Experiment}

Turbidity was used to characterize the dispersion and aggregation of mineral particles. The pyrite sample with a size range between 38 to $74 \mu \mathrm{m}$ and the serpentine sample with 104 a size of less than $10 \mu \mathrm{m}$ were weighed to $1 \mathrm{~g}$, respectively, flotation reagents were added and stirred for $5 \mathrm{~min}$, and the mix was then deposited in a $100 \mathrm{~mL}$ settling cylinder for $3 \mathrm{~min}$. A volume of $20 \mathrm{~mL}$ of the upper suspension was extracted and put into a sample bottle. The turbidity was measured using a WGZ-3(3A) scattering light turbidimeter (Xinrui Instruments, Shanghai, China). When the turbidity is high, there are more mineral particles in the upper suspension of the sedimentation system, and the pulp is in a dispersed state. On the contrary, when the turbidity is low, the mineral particles in the upper suspension are less numerous, and the pulp is in an aggregated state.

\subsubsection{Microscopic Observation}

A quantity of $2 \mathrm{~g}$ of artificial mixed ore sample (serpentine/pyrite $=1: 9$ ) was weighed, and flotation reagent of a certain concentration was added and stirred for $5 \mathrm{~min}$. A small amount of slurry droplets was removed by a needle tube under stirring conditions and placed on a slide to observe and photograph the dispersion of minerals under an Olympus CX31 transmission light microscope (Olympus Corporation, Tokyo, Japan). The image head captured the observed electronic image. Image-Pro Plus graphics software was used to analyze and process the obtained microphotographs, and the background rate of the microphotographs and the number of serpentine particles were obtained. The background rate represents the percentage of background area without particles in the image, and the larger the background rate, the smaller the proportion of mineral particles in the area, as the worse the dispersion; conversely, the smaller the background rate, the better the dispersion of mixed minerals $[26,27]$. The number of particles represents the number of mineral particles with obvious 
boundaries in the image. The smaller the number of particles, the worse the dispersion of the mixed ore. The more particles there are, the better the dispersion of the mixed ore.

\subsubsection{Zeta Potential Measurements}

The zeta potential of mineral surfaces was measured using a ZetaPALS instrument (Brookhaven Instruments, Brookhaven, MS, USA). The samples were ground to less than $5 \mu \mathrm{m}$. The suspension was prepared by adding $50 \mathrm{mg}$ of pure minerals to $5 \mathrm{~mL}$ of distilled, deionized water containing $10^{-3} \mathrm{~mol} / \mathrm{L}$ $\mathrm{KCl}$ as a supporting electrolyte. The resulting suspension was conditioned for 15 min during which the suspension $\mathrm{pH}$ was measured. The $\mathrm{pH}$ was adjusted using either $\mathrm{NaOH}$ or $\mathrm{H}_{2} \mathrm{SO}_{4}$ over the $\mathrm{pH}$ range of $2-12$.

\subsubsection{FT-IR Spectroscopy}

We weighed $2 \mathrm{~g}$ of the single-mineral sample with a particle size of less than $2 \mu \mathrm{m}$, added a certain concentration of flotation agent, stirred well, and let it stand for $30 \mathrm{~min}$. After the mineral was completely settled, the supernatant liquid was sucked out using a pipette. The mineral was thoroughly washed with distilled water and dried naturally after solid-liquid separation. Infrared spectroscopy was carried out using a Nicolet FTIR-740 Fourier-transform infrared spectrometer (Nicolet Instrument, Waltham, MA, USA).

\section{Results and Discussion}

\subsection{Effect of $\mathrm{Mg}^{2+}$ Ions on the Heterogeneous Flocculation Behavior of Pyrite and Serpentine}

Figure 2 shows the effect of $\mathrm{Mg}^{2+}$ ion dissolution on the aggregation and dispersion behavior of serpentine and pyrite. The results revealed that the dispersion of demagnesium serpentine and pyrite was improved significantly at $\mathrm{pH}>7$, and the heterogeneous coagulation between particles was weakened. When $\mathrm{Mg}^{2+}$ ions were added, the dispersion of demagnesium serpentine and pyrite decreased at $\mathrm{pH}>8$, the mineral particles re-aggregated, and the dispersion decreased. This indicated that the dispersion of serpentine and pyrite requires not only the removal of magnesium from the serpentine surface, but also the dissolution of $\mathrm{Mg}^{2+}$ ions from the pulp system.

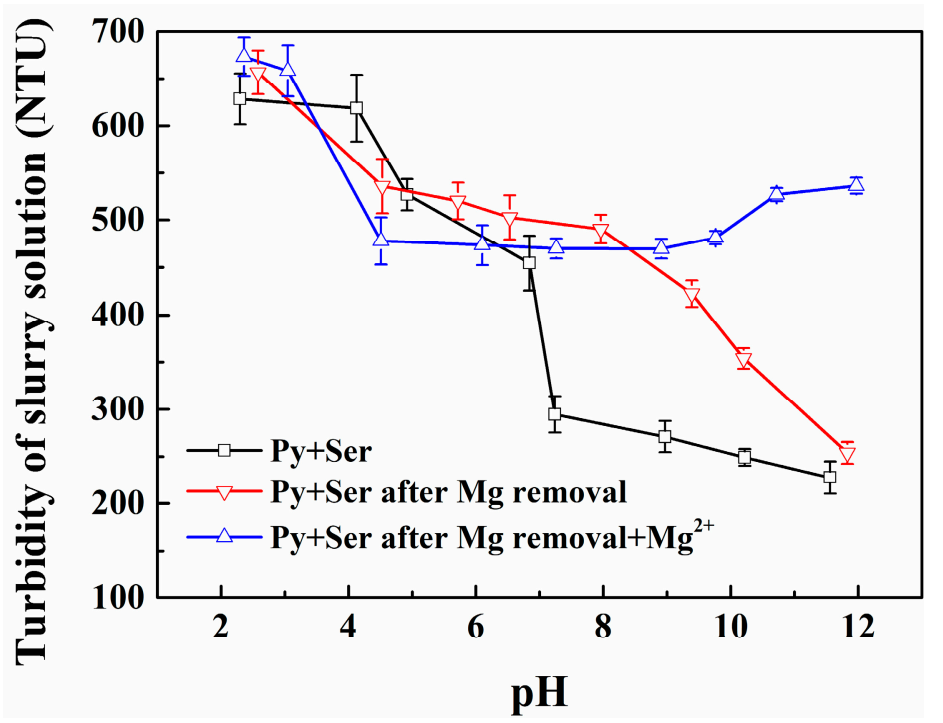

Figure 2. The effect of $\mathrm{Mg}^{2+}$ ion dissolution on the aggregation and dispersion behavior of serpentine and pyrite. ([Serpentine $]=1 \mathrm{~g} / \mathrm{L},[$ Pyrite $\left.]=9 \mathrm{~g} / \mathrm{L},\left[\mathrm{Mg}^{2+}\right]=1 \times 10^{-3} \mathrm{~mol} / \mathrm{L}\right)$. 
The dispersion of serpentine and pyrite in pulp can be observed directly by a microscope [24,28]. Figure 3 shows microscopic photos of serpentine and pyrite in the pulp. In the microscopic photos, the large black particles are pyrite and the small light grains are serpentine.
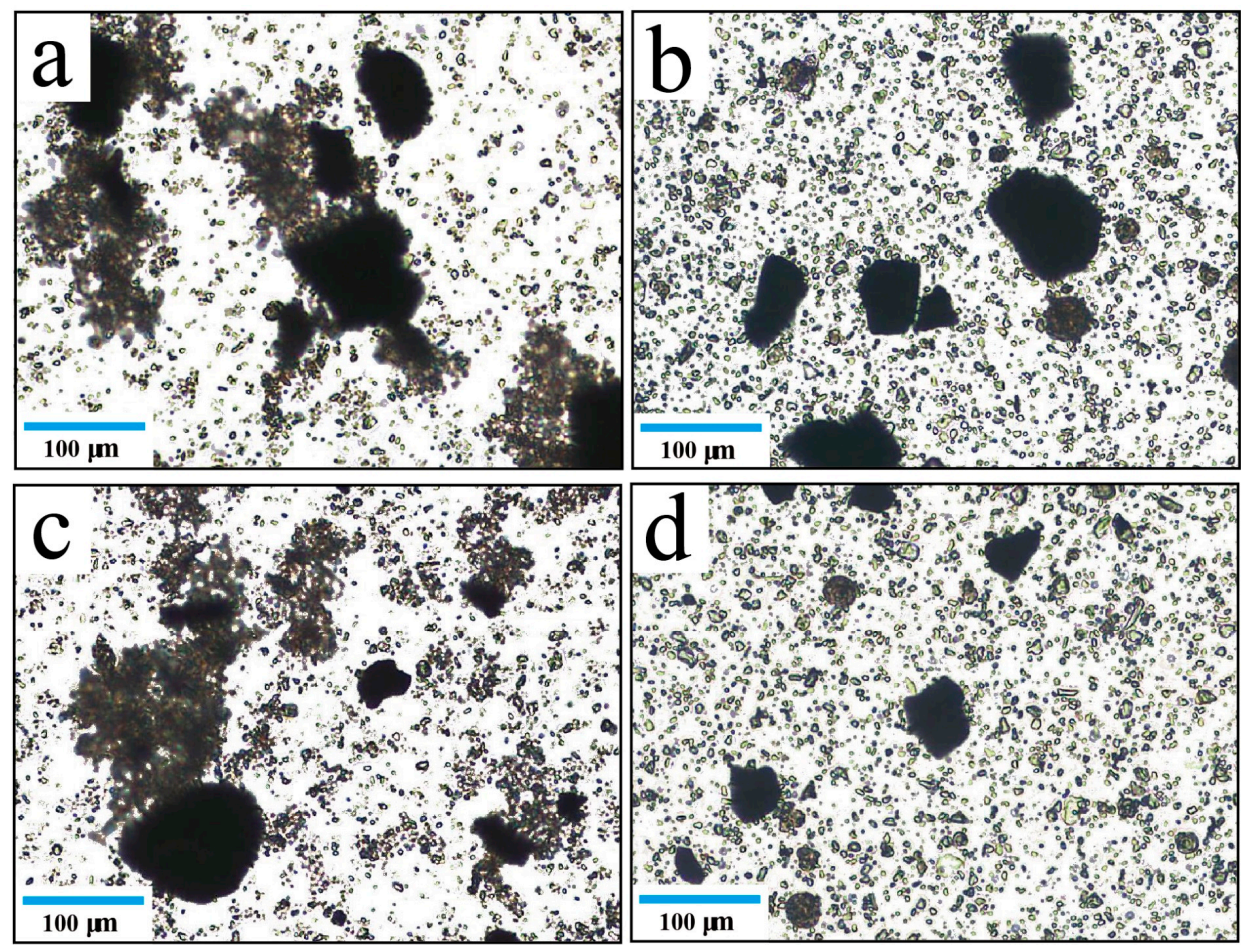

Figure 3. Microscopic photographs of serpentine and pyrite pulps treated under different conditions: (a) pyrite + serpentine; (b) pyrite + demagnesium serpentine; (c) pyrite + demagnesium serpentine + $\mathrm{Mg}^{2+}$ ions; (d) pyrite + serpentine + sodium hexametaphosphate (SHMP).

There was obvious heterogeneous agglomeration between the pyrite and serpentine particles before magnesium removal (in Figure 3a). After magnesium was removed from the serpentine surface (in Figure 3b), the dispersion of pyrite and serpentine in the pulp became better, and the heterogeneous agglomeration between particles was weakened. When $\mathrm{Mg}^{2+}$ ions were added to the pulp of pyrite and demagnesium serpentine (in Figure 3c), the particles of pyrite and serpentine re-aggregated, and the dispersion of the pulp became poorer.

Table 2 shows the background rates of the microphotographs and the numbers of serpentine particles under various experimental conditions. The smaller the number of particles, the worse the dispersion of serpentine and pyrite, and the more numerous the particles, the better the dispersion. After removing magnesium from serpentine, the background rate of the pulp solution decreased from $62.32 \%$ to $53.47 \%$, and the number of serpentine particles increased from 592 to 1188 , which indicates that $\mathrm{Mg}^{2+}$ ions on the serpentine surface seriously affected the dispersion effect between mineral particles. After adding $\mathrm{Mg}^{2+}$ ions to the demagnesium serpentine pulp, the background rate obviously increased (from 53.47\% to 58.15\%), and the number of serpentine particles decreased to 841, which indicates that the dispersion effect between mineral particles could also be affected by $\mathrm{Mg}^{2+}$ ions in solution. Comparison with Figure 3b,d shows that SHMP can also eliminate heterogeneous flocculation between pyrite and serpentine particles. The results of the background rates in pulp solution (53.47\% vs. $53.15 \%)$ and the numbers of serpentine particles (1188 vs. 1147) reveal that the dispersion of solutions after the removal of magnesium from serpentine and addition of SHMP is similar. 
Table 2. Background rate and number of serpentine particles in microscopic photographs of serpentine and pyrite.

\begin{tabular}{ccc}
\hline Sample & Background Rate (\%) & Number of Serpentine Particles \\
\hline Pyrite + serpentine & 62.32 & 592 \\
Pyrite + demagnesium serpentine & 53.47 & 1188 \\
Pyrite + demagnesium serpentine $+\mathrm{Mg}^{2+}$ & 58.15 & 841 \\
\hline Pyrite + serpentine + SHMP & 53.15 & 1147 \\
\hline
\end{tabular}

\subsection{Dissolution and Phosphate Complexation of $\mathrm{Mg}^{2+}$ Ions on the Serpentine Surface}

\subsubsection{Effect of $\mathrm{Mg}^{2+}$ Ion Dissolution on the Zeta Potential of the Serpentine Surface}

Reducing the density of localized ions in the double layer is an effective way to control the surface charge properties of particles [26,29]. As $\mathrm{Mg}^{2+}$ ions are positioning ions in a serpentine diffused double layer, reducing the concentration of $\mathrm{Mg}^{2+}$ on the serpentine surface can reduce the positive charge density of the positioning ions in the double layer, thus reducing the surface potential of serpentine.

Figure 4 shows the effect of $\mathrm{Mg}^{2+}$ ion dissolution on the zeta potential of the serpentine surface. After removing magnesium from the serpentine, the zeta potential of the serpentine shifted negatively, and the isoelectric point (IEP) decreased from a pH of 10.0 to 5.4. When $\mathrm{Mg}^{2+}$ ions $\left(1 \times 10^{-3} \mathrm{~mol} \cdot \mathrm{L}^{-1}\right)$ were added to the demagnesium serpentine slurry, the zeta potentials of the serpentine surface decreased slightly at a $\mathrm{pH}$ value below 8 , and were maintained at about $+20 \mathrm{mV}$ at a pH value above 8 . This suggests that the surface potential could recover a high positive value by the addition of $\mathrm{Mg}^{2+}$ ions. Therefore, reducing the zeta potential of serpentine requires not only the dissolution of $\mathrm{Mg}^{2+}$ ions from the mineral surface, but also the removal of dissolved $\mathrm{Mg}^{2+}$ ions from the slurry system.

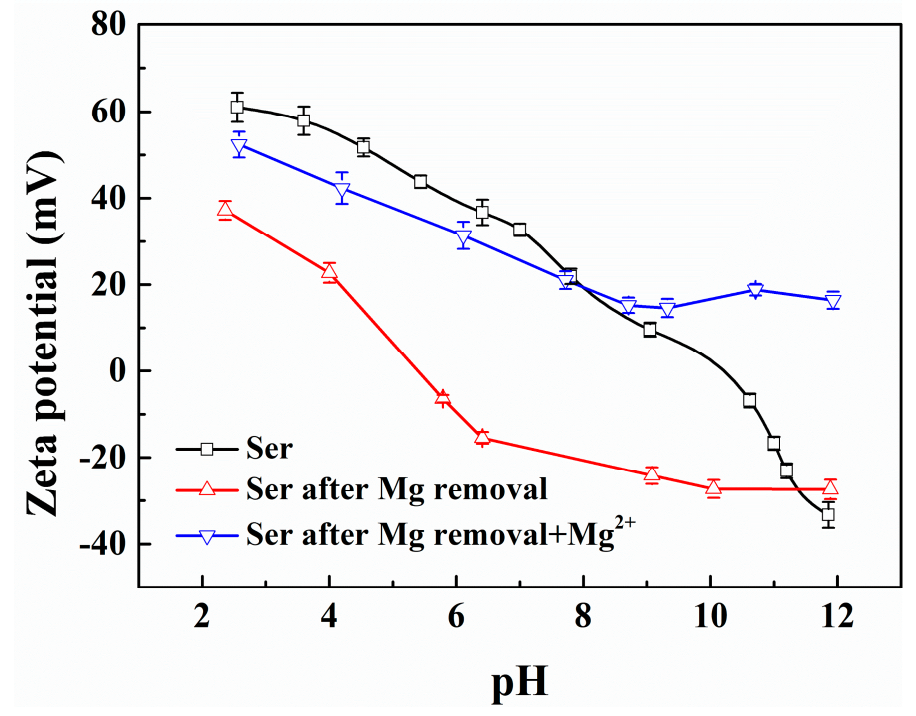

Figure 4. Zeta potential of different serpentine surfaces as a function of $\mathrm{pH}$ values. $\left(\left(\mathrm{Mg}^{2+}\right)=1 \times 10^{-3} \mathrm{~mol} / \mathrm{L}\right)$.

\subsubsection{Phosphate Complexation on Surface Potentials of Serpentine}

The complexation of $\mathrm{Mg}^{2+}$ ions on the serpentine surface can effectively regulate its surface potential and eliminate the heterogeneous condensation between serpentine and other mineral particles. On this basis, the adsorption of anion modifier on the serpentine surface further reduces its surface potential and strengthens the regulation of the surface charge properties of serpentine $[9,30]$.

Figure 5 shows the effect of three phosphates on the zeta potential of the demagnesium serpentine surface. In Figure 5a, trisodium phosphonate (TSP) showed little effect on the zeta potential of the demagnesium serpentine surface. Sodium hexametaphosphate (STMP) and sodium trimetaphosphate 
were able to significantly reduce the zeta potential of the demagnesium serpentine surface, especially SHMP. Figure $5 b$ shows that the zeta potential of demagnesium serpentine gradually decreased with increasing phosphate dosage, and finally tended to be stable. The ability of the three phosphates to reduce the zeta potential of demagnesium serpentine was in the order of SHMP > STMP > TSP.
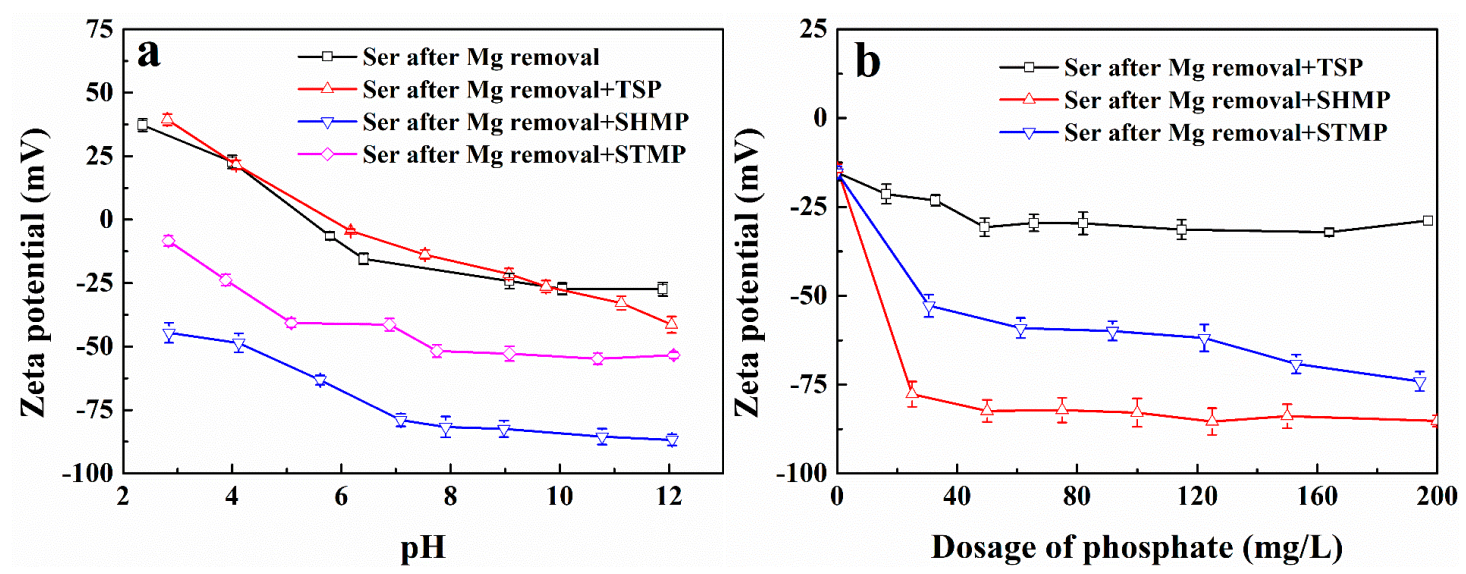

Figure 5. Zeta potentials of the serpentine surface as a function of (a) $\mathrm{pH}$ and (b) dosage of phosphate.

\subsubsection{FT-IR Spectra of Phosphate Adsorption on the Serpentine Surface}

The presence of phosphate adsorption on the serpentine surface is shown in Figure 6, which presents the infrared spectra of the phosphate and serpentine before and after phosphate adsorption. In the infrared spectrum of serpentine, the adsorption peak at $3686.0 \mathrm{~cm}^{-1}$ corresponds to the stretching vibration peak of hydroxyl groups in the serpentine magnesia-oxygen octahedron [5]. The broad and strong adsorption peak at $975.8 \mathrm{~cm}^{-1}$ is the $\mathrm{Si}-\mathrm{O}$ stretching vibration peak in the silicon-oxygen tetrahedron. The characteristic peaks of the out-of-plane bending vibration at 612.3 and $557.3 \mathrm{~cm}^{-1} \mathrm{are}^{-}$ $\mathrm{O}-\mathrm{H}$ and $\mathrm{Mg}-\mathrm{O}$, respectively. The peak at $451.1 \mathrm{~cm}^{-1}$ is the result of the coupling of the Si-O bending vibration, $\mathrm{Si}-\mathrm{O}-\mathrm{Mg}, \mathrm{Mg}-\mathrm{O}$ vibration, and $\mathrm{OH}$ translation.

In the infrared spectrum of TSP (black line in Figure 6a), the asymmetric stretching vibration and the bending vibration peaks of $\mathrm{P}-\mathrm{O}$ in $\mathrm{PO}_{3}$ correspond to 1009.7 and $551.6 \mathrm{~cm}^{-1}$, respectively. After TSP was adsorbed on the serpentine surface (red line in Figure 6a), there was no obvious TSP characteristic absorption peak, but the position of the $\mathrm{Mg}-\mathrm{O}$ characteristic absorption peak in serpentine shifted considerably (offset by more than $10 \mathrm{~cm}^{-1}$ ). In the infrared spectrum of SHMP (black line in Figure 6b), the peaks at $1279.9,1096.8$, and $875 \mathrm{~cm}^{-1}$ correspond to the $\mathrm{P}-\mathrm{O}$ stretching vibration, and the $\mathrm{P}-\mathrm{O}$ bending vibration peak is located at $515.4 \mathrm{~cm}^{-1}$ [31]. In the infrared spectrum of STMP (black line in Figure $6 \mathrm{c}$ ), the peaks at 1297.0 and $997.9 \mathrm{~cm}^{-1}$ correspond to the $\mathrm{P}-\mathrm{O}$ stretching vibration, and the $\mathrm{P}-\mathrm{O}$ bending vibration peak is located at $519.2 \mathrm{~cm}^{-1}$. The infrared spectra of the serpentine surface after SHMP and STMP adsorption are similar to that of TSP adsorption. Similarly, the P-O characteristic peak was not found, and the $\mathrm{Mg}-\mathrm{O}$ characteristic peak in serpentine also showed a large displacement. This indicated that all three phosphates were chemically adsorbed on the serpentine surface, but their adsorption capacity was very little. 

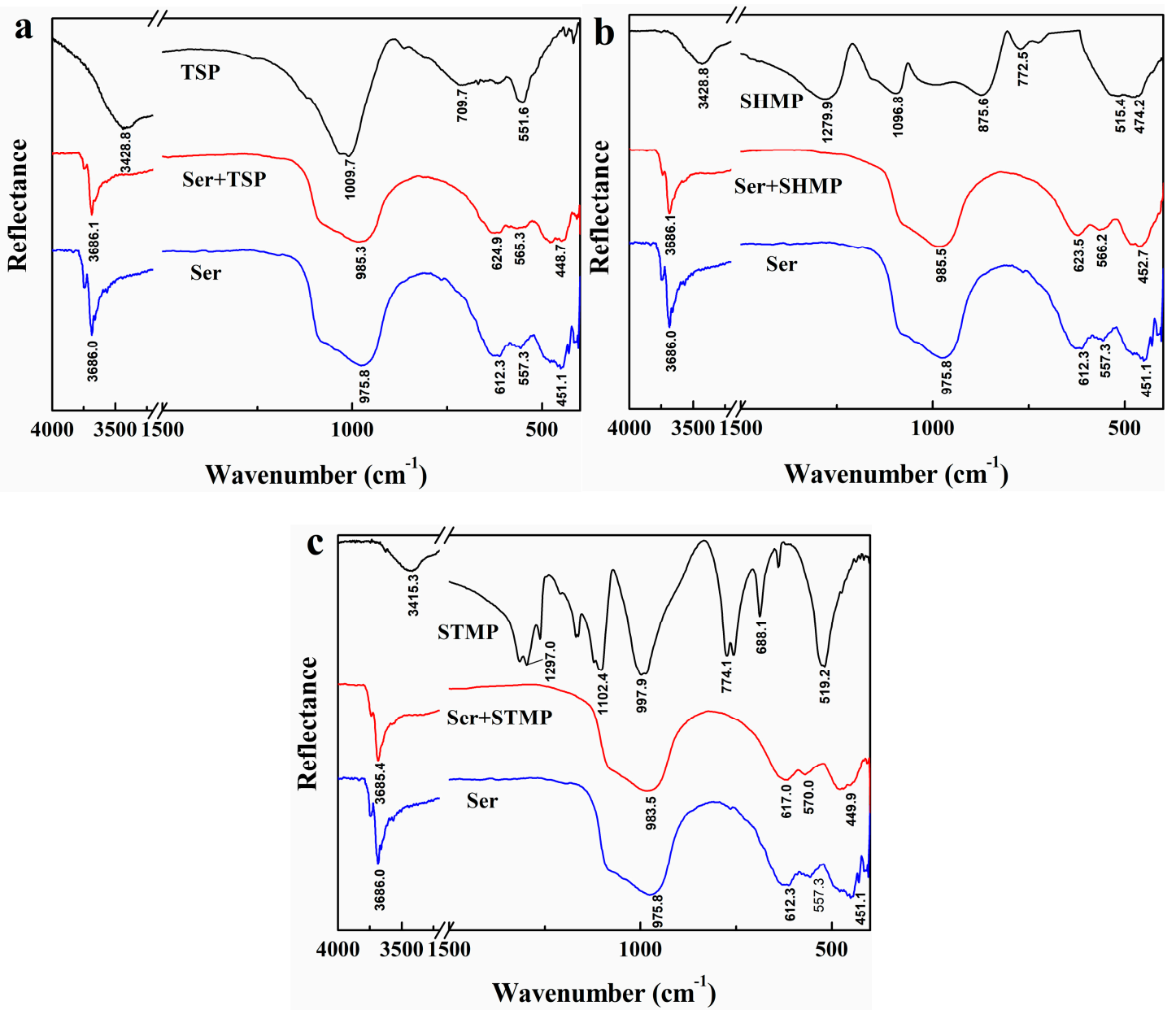

Figure 6. FT-IR spectra of the serpentine surface before and after different phosphate treatments: (a) trisodium phosphonate (TSP); (b) SHMP; (c) sodium trimetaphosphate (STMP).

\subsection{Effect of Surface Charge on the Interaction Energy between Mineral Particles}

According to the classical DLVO theory, the total potential energy $V_{T}$ of particle interaction in heterogeneous mineral water-based suspensions is derived by Equation (1) [32]:

$$
V_{T}=V_{W}+V_{E}
$$

where $V_{W}$ is the Van der Waals interaction energy and $V_{E}$ is the electrostatic interaction energy.

The Van der Waals interaction between spherical particles can be governed by Equation (2):

$$
V_{w}=-\frac{A}{6 H} \frac{R_{1} R_{2}}{R_{1}+R_{2}}
$$

where $A$ is the effective Hamaker constant of Minerals 1 and 2 in a water medium, $R_{1}$ is the radius of a Mineral 1 spherical particle, $R_{2}$ is the radius of a Mineral 2 spherical particle, and $\mathrm{H}$ is the distance between Mineral 1 and Mineral 2 particles. The expression of $A$ is [33]

$$
A=\left(\sqrt{A_{11}}-\sqrt{A_{33}}\right)\left(\sqrt{A_{22}}-\sqrt{A_{33}}\right)
$$

where $A_{11}$ and $A_{22}$ are the Hammaker constants of Mineral 1 and 2 in vacuum, respectively, and $A_{33}$ is the Hammaker constant of water in vacuum $\left(A_{33}=3.7 \times 10^{-20} \mathrm{~J}\right)[34]$. 
The expression of the electrostatic interaction energy $V_{E}$ between the particles of Mineral 1 and Mineral 2 is as follows:

$$
V_{E}=\frac{\pi \varepsilon_{0} \varepsilon_{r} R_{1} R_{2}}{R_{1}+R_{2}}\left(\psi_{1}^{2}+\psi_{2}^{2}\right)\left\{\frac{2 \psi_{1} \psi_{2}}{\left(\psi_{1}^{2}+\psi_{2}^{2}\right)} \ln \left[\frac{1+\exp (-k H)}{1-\exp (-k H)}\right]+\ln [1-\exp (-2 k H)]\right.
$$

where $\varepsilon_{0}$ is the absolute dielectric constant in vacuum $\left(\varepsilon_{0}=8.854 \times 10^{-12}\right) ; \varepsilon_{r}$ is the dielectric constant of the dispersing medium (water, $\varepsilon_{r}=78.5 \mathrm{C}^{2} \cdot \mathrm{J}^{-1} \cdot \mathrm{m}^{-1}$ ); $\psi_{1}$ and $\psi_{2}$ are the surface potentials of Mineral 1 and Mineral 2 particles, respectively; $\zeta$ is the zeta potential of the mineral solid-liquid interface; $x$ is the distance from the surface of charged mineral particles to the slip surface $\left(x=5 \times 10^{-10} \mathrm{~m}\right) ;$ and $\kappa^{-1}$ is the Debye length, representing the thickness of the double layer.

$\psi$ and $\kappa$ are obtained by Equations (5) and (6), respectively:

$$
\begin{gathered}
\psi=\zeta(1+x / R) \exp (k x) \\
k=\left(\frac{4 e^{2} N_{A} I}{\varepsilon_{0} \varepsilon_{r} K_{B} T}\right)^{1 / 2}
\end{gathered}
$$

where $e$ is the electron charge $\left(e=1.602 \times 10^{-19} \mathrm{C}\right) ; N_{A}$ is the Avogadro constant $\left(N_{A}=6.02 \times 10^{23}\right) ; I$ is the ionic strength of the solution $\left(I=1 \times 10^{-3} \mathrm{~mol} / \mathrm{L}\right) ; K_{B}$ is the Boltzmann constant $\left(K_{B}=1.38 \times 10^{-23}\right.$ $\mathrm{J} / \mathrm{K})$; and $T$ is the thermodynamic temperature $(T=298 \mathrm{~K})$.

In the aqueous suspension system of serpentine and pyrite, $A_{11}=9.7 \times 10^{-20} \mathrm{~J}, A_{22}=1.2 \times 10^{-19}$ $\mathrm{J}$ [35], $R_{1}=3.09 \times 10^{-6} \mathrm{~m}$, and $R_{2}=2.67 \times 10^{-5} \mathrm{~m}$. By synthesizing Equations (1) $-(6)$, the following can be concluded:

$$
\begin{aligned}
V_{T}=V_{w}+V_{E}= & -8.45 \times 10^{-27} / H \\
& +7.0 \times 10^{-21} \times\left\{\left(\zeta_{1}+\zeta_{2}\right)^{2} \ln [1+\exp (-0.1467 H)]\right. \\
& \left.+\left(\zeta_{1}-\zeta_{2}\right)^{2} \ln [1-\exp (-0.1467 H)]\right\}
\end{aligned}
$$

The flotation of copper-nickel sulphide ores is generally carried out under conditions of a weak alkaline $\mathrm{pH}$. For a pulp $\mathrm{pH}$ of 9.0 , the results of the zeta potentials of the serpentine and pyrite surfaces are shown in Table 3.

Table 3. Zeta potential of mineral surface under different conditions.

\begin{tabular}{cccc}
\hline \multirow{2}{*}{ Mineral } & \multicolumn{3}{c}{ Zeta Potential (mV) } \\
\cline { 2 - 4 } & Original & Demagnesium & SHMP \\
\hline Serpentine & 9.58 & -24.25 & -59.73 \\
Pyrite & -44.35 & -44.35 & -65.26 \\
\hline
\end{tabular}

According to the results in Table 3, the relationship between the total potential energy of interaction between serpentine and pyrite particles in the water medium and the distance between particles is shown in Figure 7 . When the $\mathrm{pH}$ is 9 , the interaction energy between serpentine and pyrite particles is negative. The interaction force between serpentine and pyrite particles shows mutual attraction (in Curve 1). With decreasing particle spacing, the attraction gradually increases and heterogeneous agglomeration easily occurs. The interaction energy between serpentine and pyrite becomes positive, the interaction force between serpentine and pyrite particles is mutually exclusive, and the dispersion between serpentine and pyrite particles is improved (in Curve 2). When sodium hexametaphosphate is added, the interaction energy between serpentine and pyrite is positive, and the interaction force between particles is mutually exclusive. At this time, the exclusion energy between particles is greater than that between serpentine and pyrite after magnesium removal (in Curve 3). Serpentine and pyrite can maintain a better dispersion state in the system. 


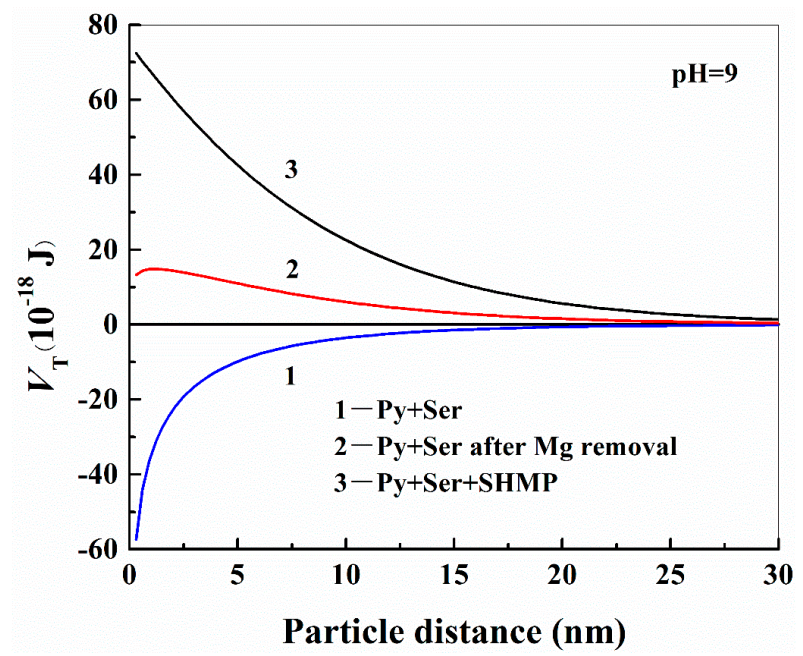

Figure 7. Relation between total potential energy of interaction between serpentine and pyrite particles in aqueous media and particle spacing.

\subsection{Effect of Surface Potential Regulation on the Flotation Separation of Pyrite and Serpentine}

The effect of surface potential regulation on the flotation separation of pyrite and serpentine was investigated by a flotation test of artificial mixed ore, as shown in Figure 8. In Figure 8a, we can see that the single mineral of pyrite had good floatability at $\mathrm{pH}<10$, and the flotation recovery was able to reach more than $90 \%$. The addition of serpentine significantly reduced the flotation recovery of pyrite. After removing magnesium from the serpentine, the flotation recovery of pyrite in the artificial mixed ore increased, and the floatability of pyrite was restored. After removing magnesium from the serpentine and adding $\mathrm{Mg}^{2+}$ ions, the flotation recovery of pyrite in the artificial mixed ore decreased rapidly at $\mathrm{pH}>8$, and the floatability of pyrite was affected by serpentine again. As the surface charge properties of serpentine can be significantly reduced by chain polyphosphate, the effects of sodium pyrophosphate, sodium tripolyphosphate, and sodium hexametaphosphate on the flotation separation of serpentine and pyrite were investigated, as shown in Figure 8b. The effect of chain polyphosphates on the flotation of artificial mixed ores was assessed under different $\mathrm{pH}$ values. It can be seen from the graph that all three kinds of polyphosphates were able to improve the flotation recovery of pyrite in the mixed ore, restore the floatability of pyrite, and eliminate the influence of serpentine on the flotation of pyrite.
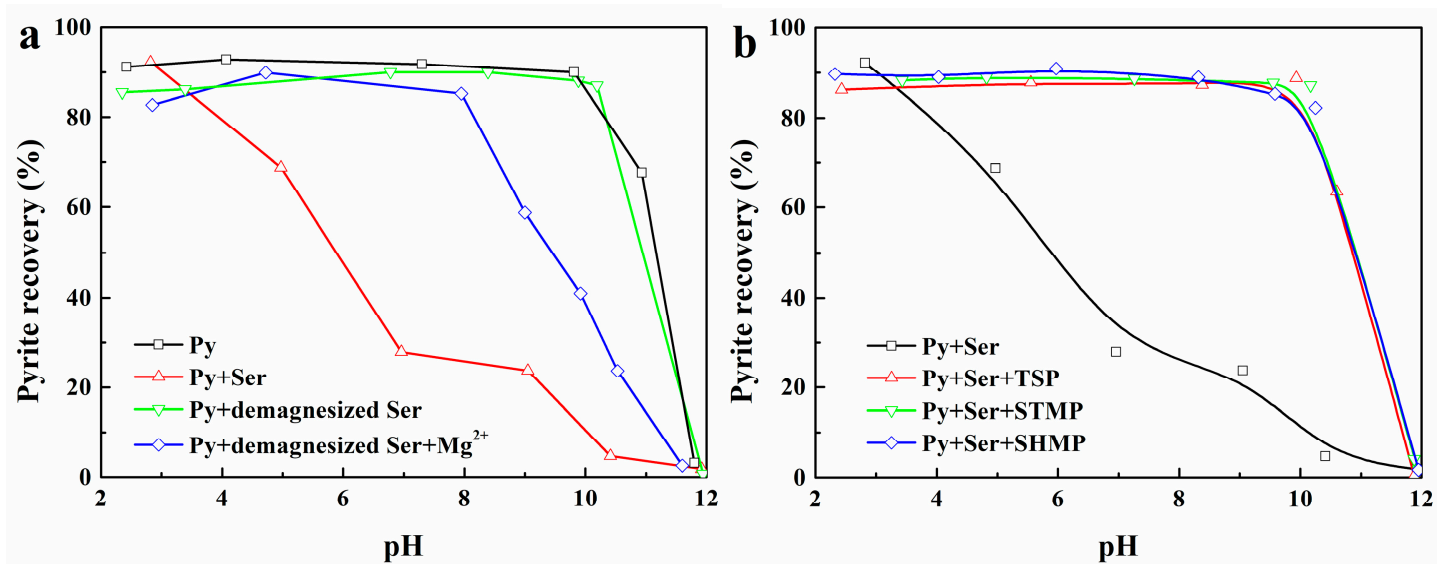

Figure 8. Effect of surface potential regulation of the flotation separation of pyrite and serpentine: (a) removal of magnesium from the serpentine surface; (b) addition of different phosphates. 


\subsection{Mechanism Model of Surface Potential Regulation on the Serpentine Surface}

Serpentine belongs to the TO-type layered silicate minerals, and its structural unit layer is composed of magnesia-oxygen octahedrons and silica-oxygen tetrahedrons. Serpentine dissociation exposes the magnesia-oxygen octahedral layer in the crystal structure, $\mathrm{OH}$-preferential dissociation occurs in the serpentine-magnesia-oxygen octahedral in aqueous solution, and residual magnesium is exposed on the surface, which makes the serpentine surface positively charged. $\mathrm{Mg}^{2+}$ ions are one of the positioning ions in the serpentine double layer. Removal of $\mathrm{Mg}^{2+}$ ions from the serpentine surface can reduce the positive charge density of positioning ions in the double layer, thus reducing the surface potential of serpentine. Figure 9 is a schematic diagram showing that the $\mathrm{Mg}^{2+}$ ion dissolution and phosphate complexation reduce the surface potential of serpentine. The removal of magnesium ions from the serpentine surface reduces the positive charge density of positioning ions in the serpentine double layer, thereby reducing the surface charge properties of serpentine. The reverse adsorption of magnesium ions to the serpentine surface in solution will re-increase the positioning in the double layer. The positive charge density of ions then increases the surface charge properties of the serpentine. Therefore, the surface potential of serpentine can be regulated by the $\mathrm{Mg}^{2+}$ ion dissolution on the serpentine surface. On the one hand, magnesium ions should be transferred from the serpentine surface to solution; on the other hand, the dissolved $\mathrm{Mg}^{2+}$ ions can be prevented from re-entering the double layer of the serpentine solid-liquid interface. Sodium hexametaphosphate is easily hydrolyzed in aqueous solution to form polyphosphate anions. In the pulp, these anions are adsorbed on the serpentine surface by chemical interaction with the metal particles onto the serpentine surface, which causes a negative charge on the serpentine surface.

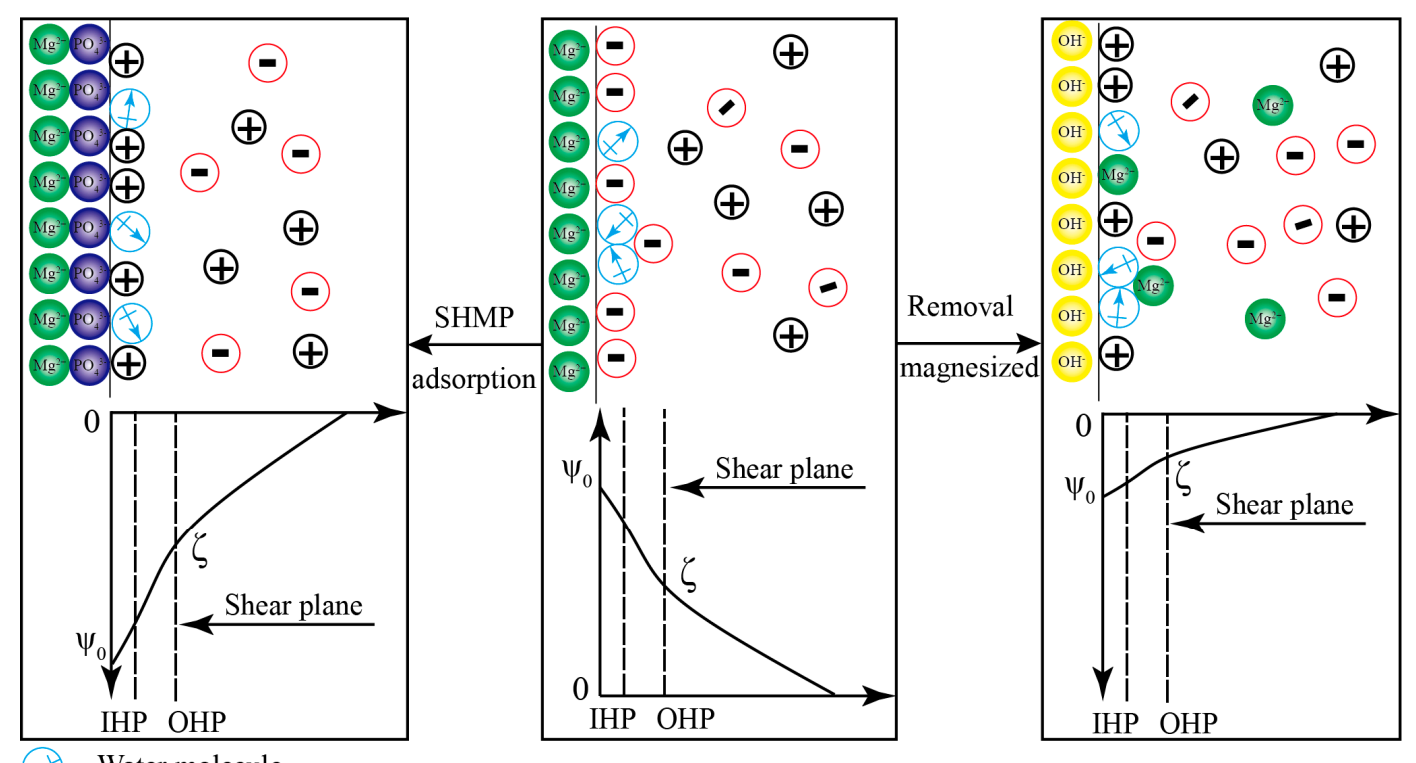

Figure 9. Schematic diagram of the surface potential regulation of serpentine by $\mathrm{Mg}^{2+}$ ion dissolution and phosphate complexation. (In the middle is the original serpentine surface double layer. In order to reduce the surface potential of serpentine, on the left side, the surface of serpentine is complexed by adding phosphates, and on the right side, the surface of serpentine is treated by pickling.).

\section{Conclusions}

In summary, we investigated the effect of the surface charge regulation of serpentine on mineral flotation, and we studied the mechanism of $\mathrm{Mg}^{2+}$ ion dissolution and complexation from the serpentine surface to liquid and its effect on its surface charge properties. The following conclusions were drawn:

(1) Strengthened $\mathrm{Mg}^{2+}$ ion dissolution from the serpentine surface to the liquid phase can reduce the positive charge density of $\mathrm{Mg}^{2+}$ positioning ions in the double layer. This reduces the surface 
potential of serpentine, thereby eliminating heterogeneous condensation between serpentine and pyrite, and achieving good dispersion between mineral particles.

(2) Chain-like polyphosphates can reduce the surface potential of serpentine. The mechanism mainly includes three aspects: They can enhance the dissolution of $\mathrm{Mg}^{2+}$ ions from the serpentine surface to the liquid phase and reduce the surface potential of serpentine; they can interact with $\mathrm{Mg}^{2+}$ in the liquid phase to form a stable soluble complex, thus maintaining the surface of serpentine; and the surface potential of serpentine is further reduced by adsorption onto the surface of serpentine.

(3) Under natural conditions, the interfacial interaction between serpentine and pyrite in aqueous solution shows mutual attraction and heterogeneous coagulation between mineral particles. After adjusting the surface charge properties of serpentine, the interfacial interaction between serpentine and pyrite particles showed mutual exclusion, and mineral particles were dispersed in the solution system.

(4) After adjusting the surface charge properties of serpentine, the dispersion of mineral particles was improved, and the artificial mixture of pyrite and serpentine could be effectively separated by flotation.

Author Contributions: W.X. conceived of and designed the experiments; T.L. prepared the samples and performed the experiments; X.H. and T.L. analyzed the data. W.X., T.L., and X.H. contributed to the writing and revising of the paper.

Funding: The authors acknowledge the Project Supported by the National Natural Science Foundation of China (Nos. 51304150 and 51474169).

Conflicts of Interest: The authors declare no conflict of interest.

\section{References}

1. Rosenkranz, T.; Hipfinger, C.; Ridard, C.; Puschenreiter, M. A nickel phytomining field trial using Odontarrhena chalcidica and Noccaea goesingensis on an Austrian serpentine soil. J. Environ. Manag. 2019, 242, 522-528. [CrossRef]

2. Yang, W.; Wang, G.; Wang, Q.; Dong, P.; Cao, H.; Zhang, K. Comprehensive recovery technology for Te, Au, and Ag from a telluride-type refractory gold mine. Minerals 2019, 9, 597. [CrossRef]

3. Wu, S.; Ramandi, H.L.; Chen, H.; Crosky, A.; Hagan, P.; Saydam, S. Mineralogically influenced stress corrosion cracking of rockbolts and cable bolts in underground mines. Int. J. Rock Mech. Min. Sci. 2019, 119, 109-116. [CrossRef]

4. Mobarak, M.; Mohamed, E.A.; Selim, A.Q.; Sellaoui, L.; Lamine, A.B.; Erto, A.; Bonilla-Petriciolet, A.; Seliem, M.K. Surfactant-modified serpentine for fluoride and $\mathrm{Cr}(\mathrm{VI})$ adsorption in single and binary systems: Experimental studies and theoretical modeling. Chem. Eng. J. 2019, 369, 333-343. [CrossRef]

5. Li, Z.; Han, Y.; Li, Y.; Gao, P. Effect of serpentine and sodium hexametaphosphate on ascharite flotation. Trans. Nonferrous Met. Soc. China 2017, 27, 1841-1848. [CrossRef]

6. Alvarez-Silva, M.; Uribe-Salas, A.; Waters, K.E.; Finch, J.A. Zeta potential study of pentlandite in the presence of serpentine and dissolved mineral species. Miner. Eng. 2016, 85, 66-71. [CrossRef]

7. Zhao, H.; Zhang, Y.; Zhang, X.; Qian, L.; Sun, M.; Yang, Y.; Zhang, Y.; Wang, J.; Kim, H.; Qiu, G. The dissolution and passivation mechanism of chalcopyrite in bioleaching: An overview. Miner. Eng. 2019, 136, 140-154. [CrossRef]

8. Long, T.; Xiao, W.; Yang, W. The effect of molecular assembly between collectors and inhibitors on the flotation of pyrite and talc. R. Soc. Open Sci. 2019, 6, 191133. [CrossRef]

9. Xiao, W.; Ren, Y.; Yang, J.; Cao, P.; Wang, J.; Qin, W.; Qiu, G. Adsorption mechanism of sodium oleate and styryl phosphonic acid on rutile and amphibole surfaces. Trans. Nonferrous Met. Soc. China 2019, 29, 1939-1947. [CrossRef]

10. Yang, W.; Wang, Q.; Wang, Y.; Dong, P. Eliminating the adverse effect of the lime on the gold-bearing pyrrhotite flotation using the isopentyl xanthate as collector at low alkalinity. Physicochem. Probl. Miner. Process. 2019, 55, 1250-1258. 
11. Zhang, C.; Liu, C.; Feng, Q.; Chen, Y. Utilization of N-carboxymethyl chitosan as selective depressants for serpentine on the flotation of pyrite. Int. J. Miner. Process. 2017, 163, 45-47. [CrossRef]

12. Zhou, X.; Feng, B. The effect of polyether on the separation of pentlandite and serpentine. J. Mater. Res. Technol. 2015, 4, 429-433. [CrossRef]

13. Lu, J.; Yuan, Z.; Li, M.; Zhao, X.; Tong, Z.; Li, L.; Qi, S. The role of sodium oleate (NaOL) in the magnetic separation of pentlandite from serpentine using magnetic coating. Powder Technol. 2019, 345, 492-500. [CrossRef]

14. Yang, S.; Xie, B.; Lu, Y.; Li, C. Role of magnesium-bearing silicates in the flotation of pyrite in the presence of serpentine slimes. Powder Technol. 2018, 332, 1-7. [CrossRef]

15. Yuan, Z.; Lu, J.; Liu, J.; Li, L.; Wang, S. Enhancement of pentlandite surface magnetism and implications for its separation from serpentine via magnetic separation. Trans. Nonferrous Met. Soc. China 2017, 27, 204-210. [CrossRef]

16. Wan, H.; Yang, W.; Cao, W.; He, T.; Liu, Y.; Yang, J.; Guo, L.; Peng, Y. The Interaction between $\mathrm{Ca}^{2+}$ and Molybdenite Edges and Its Effect on Molybdenum Flotation. Minerals 2017, 7, 141. [CrossRef]

17. Ucbas, Y.; Bozkurt, V.; Bilir, K.; Ipek, H. Separation of chromite from serpentine in fine sizes using magnetic carrier. Sep. Sci. Technol. 2014, 49, 946-956. [CrossRef]

18. Nie, X.; Feng, S.; Shudu, Z.; Quan, G. Simulation study on the dynamic ventilation control of single head roadway in high-altitude mine based on thermal comfort. Adv. Civ. Eng. 2019, 2019, 12. [CrossRef]

19. Nie, X.; Wei, X.; Li, X.; Lu, C. Heat treatment and ventilation optimization in a deep mine. Adv. Civ. Eng. 2018, 2018, 1-12. [CrossRef]

20. Lu, J.; Sun, M.; Yuan, Z.; Qi, S.; Tong, Z.; Li, L.; Meng, Q. Innovative insight for sodium hexametaphosphate interaction with serpentine. Colloids Surf. A-Physicochem. Eng. Asp. 2019, 560, 35-41. [CrossRef]

21. Zhao, K.; Yan, W.; Wang, X.; Gu, G.; Deng, J.; Zhou, X.; Hui, B. Dispersive effect of low molecular weight sodium polyacrylate on pyrite-serpentine flotation system. Physicochem. Probl. Miner. Process. 2017, 53, 1200-1213.

22. Xiao, W.; Cao, P.; Liang, Q.; Huang, X.; Li, K.; Zhang, Y.; Qin, W.; Qiu, G.; Wang, J. Adsorption behavior and mechanism of $\mathrm{Bi}(\mathrm{III})$ ions on rutile-water interface in the presence of nonyl hydroxamic acid. Trans. Nonferrous Met. Soc. China 2018, 28, 348-355. [CrossRef]

23. Xiao, W.; Cao, P.; Liang, Q.; Peng, H.; Zhao, H.; Qin, W.; Qiu, G.; Wang, J. The activation mechanism of Bi ${ }^{3+}$ ions to rutile flotation in a strong acidic environment. Minerals 2017, 7, 113. [CrossRef]

24. Xiao, W.; Zhao, Y.; Yang, J.; Ren, Y.; Yang, W.; Huang, X.; Zhang, L. Effect of sodium oleate on the adsorption morphology and mechanism of nanobubbles on the mica surface. Langmuir 2019, 35, 9239-9245. [CrossRef]

25. Xiao, W.; Wang, X.; Zhou, L.; Zhou, W.; Wang, J.; Qin, W.; Qiu, G.; Hu, J.; Zhang, L. Influence of Mixing and Nanosolids on the Formation of Nanobubbles. J. Phys. Chem. B 2019, 123, 317-323. [CrossRef]

26. He, T.; Li, H.; Jin, J.; Peng, Y.; Wang, Y.; Wan, H. Improving fine molybdenite flotation using a combination of aliphatic hydrocarbon oil and polycyclic aromatic hydrocarbon. Results Phys. 2019, 12, 1050-1055. [CrossRef]

27. Li, H.; He, T.; Wang, Y.; Jin, J.; Yuan, H. XRD and SEM Analyses of Molybdenite with Different Particle Sizes and Its Floatability Difference. Spectrosc. Spectr. Anal. 2018, 38, 3588-3592.

28. Huang, X.; Xiao, W.; Zhao, H.; Cao, P.; Hu, Q.; Qin, W.; Zhang, Y.; Qiu, G.; Wang, J. Hydrophobic flocculation flotation of rutile fines in presence of styryl phosphonic acid. Trans. Nonferrous Met. Soc. China 2018, 28, 1424-1432. [CrossRef]

29. Huang, X.; Zhu, T.; Duan, W.; Liang, S.; Li, G.; Xiao, W. Comparative studies on catalytic mechanisms for natural chalcopyrite-induced Fenton oxidation: Effect of chalcopyrite type. J. Hazard. Mater. 2020, 381, 120998. [CrossRef]

30. Wang, S.; Xiao, W.; Ma, X.; Li, J.; Chen, L.; Yao, H.J.M. Analysis of the application potential of coffee oil as an ilmenite flotation collector. Minerals 2019, 9, 505. [CrossRef]

31. Liu, Q.; Feng, X.; He, Y.; Lu, C.; Gu, Q. Three-dimensional multiple-relaxation-time lattice Boltzmann models for single-phase and solid-liquid phase-change heat transfer in porous media at the REV scale. Appl. Therm. Eng. 2019, 152, 319-337. [CrossRef]

32. Bremmell, K.E.; Fornasiero, D.; Ralston, J. Pentlandite-lizardite interactions and implications for their separation by flotation. Colloids Surf. A Physicochem. Eng. Asp. 2005, 252, 207-212. [CrossRef]

33. Feng, B.; Lu, Y.; Luo, X. The effect of quartz on the flotation of pyrite depressed by serpentine. J. Mater. Res. Technol. 2015, 4, 8-13. [CrossRef] 
34. Elkin, A.P. Theoretical analysis of forces responsible for the interaction of lipid bilayer membranes in the contact zone. Biofizika 1977, 22, 444-447.

35. Wang, J.; Gan, X.; Zhao, H.; Hu, M.; Li, K.; Qin, W.; Qiu, G. Dissolution and passivation mechanisms of chalcopyrite during bioleaching: DFT calculation, XPS and electrochemistry analysis. Miner. Eng. 2016, 98, 264-278. [CrossRef] 